\title{
Locally Resonant Band Gaps in Flexural Vibrations of a Timoshenko Beam with Periodically Attached Multioscillators
}

\author{
Zhenyu Wang, Pei Zhang, and Yongqiang Zhang \\ College of Civil Engineering and Architecture, Zhejiang University, Hangzhou 310058, China \\ Correspondence should be addressed to Zhenyu Wang; wzyu@zju.edu.cn
}

Received 22 October 2012; Revised 27 January 2013; Accepted 27 January 2013

Academic Editor: Zhongqing Su

Copyright ( 2013 Zhenyu Wang et al. This is an open access article distributed under the Creative Commons Attribution License, which permits unrestricted use, distribution, and reproduction in any medium, provided the original work is properly cited.

\begin{abstract}
A new beam structure with periodically attached multioscillators is proposed based on the idea of locally resonant (LR) phononic crystals (PCs) to reduce flexural vibrations in the frequency-multiplication ranges. Wave band structures of the new beam are derived by using the transfer matrix method. The multiple band gaps in the beam are then verified by the frequency response function (FRF), which is calculated through the finite element method. In addition, simplified models are proposed, which contribute to the calculation of the edge frequencies of the band gaps and enhance the understanding of the LR mechanism of PCs. The accuracy of the simplified models is proven by comparing them with the results derived from the analytical model under different beam structure parameters. The results suggest that lower frequencies and ranges of frequency multiplications can be achieved in the band gaps which are obtained from the new beam structure with multioscillators in a unit cell. Therefore, the ideas presented in this paper have the potential to be used in developing new devices with frequency-multiplication characteristics for vibration isolation or noise control in aerospace and civil structures.
\end{abstract}

\section{Introduction}

Methods to control the propagation of elastic waves, such as vibration reduction and noise isolation, are often the focus of engineering studies. Much research has been conducted over many years to suppress unwanted vibration or noise. A variety of vibration control technologies, including visco-elastic materials, springs, soft materials, hydraulic dampers, and pneumatic isolators, among others, were gradually developed and are widely used in engineering practice [1]. As technology progresses, scientific equipment and structures are developed further to be more complex and precise. The control of the higher-order vibration or coupled vibration in these complicated structures, as well as the higher precision and flexibility of the vibration isolation in precise instruments, is increasingly important. Currently, the traditional vibration/noise control technologies are facing new challenges.

In the last decade, the emergence and development of phononic crystals (PCs) have inspired new ideas for wave control [2-4]. PCs are artificial composite materials that are formed by periodic variations of properties and structures of the material. One notable aspect of these PCs is the wave filtering property of the so-called "band gaps," which are selected frequency ranges in which elastic waves cannot propagate through the periodic system. This property means that the vibration can be well mitigated when its frequency is located in the specified band gaps of the PCs. In addition, the frequency-space distributions of the band gaps for a PC can be regulated by the properties, geometries, and arrangements of the elements composing the so-called "artificial crystal." Such a unique property promises an enormous potential for the development of vibration isolation structures [5], wave filters [6], sonic shields [7], and other applications; these developments may provide new ways to achieve the aims that are difficult to realise with the traditional vibration/noise control technologies.

There has been a great deal of research on the mechanisms and properties of band gaps. The earlier investigations of PCs are commonly based on the Bragg scattering mechanism [3]. Such band gaps are called Bragg-type gaps, whose centre frequencies are governed by the Bragg condition $a=$ $n(\lambda / 2)(n=1,2,3, \ldots)$, where $a$ is the lattice constant of the periodic system and $\lambda$ is the wavelength in the host material. The Bragg condition indicates that Bragg-type gaps 
are not practical for filtering waves in the low frequency range because the lattice constant must be of the same order as the relevant wavelength. In contrast, the locally resonant (LR) mechanism proposed by Liu et al. [8] makes it possible to obtain resonance-type gaps with lattice constants that are two orders of magnitude smaller than the relevant wavelength by proposing a type of LR PC, which has attracted considerable attention in this field [9-16]. The LR mechanism is mainly based on the idea of mounting periodic arrays of local resonators to a host medium. Thus, the frequency can be tuned to the desired values by varying the parameters and structures of the local resonators. In analogy with LR PCs, the idea of resonance-type band gaps has recently been attempted, both theoretically and experimentally, for rods [17-19], beams [5, 20-24], pipes [25], and plates [26-28] in vibration-control engineering.

Beams are typical structural elements of many engineering constructions and equipments. The control of wave propagation in beams is of great importance in aerospace and civil structures because the unwanted transmission of waves can lead to safety issues or environmental consequences. Based on the concept of LR PCs, some research focuses on the existence of low-frequency resonance gaps in infinite systems and the validation of gap characteristics by calculating/measuring the frequency response functions (FRFs) of finite samples [5, 20, 21]. Yu et al. investigated the flexural vibration band gaps in beams with locally resonant structures that have a single degree of freedom [5] and two degrees of freedom [20]. Liu et al. discussed the frequency range and attenuation coefficient of the locally resonant gap with different local resonators [21]. However, these studies all focused on a single band gap, which is not suitable for the reduction of vibrations in the multiple frequency ranges in engineering because the high-order modes of beams may also be involved in the vibrations. The same is true in a rotor system, in which the flexural vibration is also increased at two and four times the fundamental frequency due to angular misalignment of the coupling [29].

Recently, the coexistence of resonance-type and Braggtype band gaps was found in LR beams [22, 23]. Liu and Hussein observed the transition state between resonancetype and Bragg-type band gaps as well as an interesting wave behaviour caused by the interplay of these two mechanisms in LR beams [22]. Xiao et al. achieved broader band gaps in a locally resonant beam with multiple arrays of damped resonators at frequencies both below and around the Bragg condition [23]. These researchers' works can derive multiple band gaps under certain circumstances, whereas the wave attenuation in a Bragg-type band gap is too small to meet the higher isolation demand. In addition, complicated structure constructions are needed to achieve a significant amount of wave attenuation in the Bragg-type band gap. Similar research can be found in the study by Wen et al., which attempted to add multiple oscillators to a unit cell of an EulerBernoulli beam to obtain multiple resonance-type band gaps [24]. However, the first gap these researchers derived was too narrow to mitigate vibration in the low-frequency range, and the band gaps in the frequency-multiplication ranges were not provided. Thus, their research cannot well deal with the problem of vibration reduction within multiple frequency ranges, especially in the frequency-multiplication ranges.

The main purpose of this paper is to achieve more flexible resonance-type multiband gaps by proposing a new beam with periodically attached multioscillators. The lower initial frequency and band gaps in the frequency-multiplication ranges are expected to be obtained in the new beam, which can meet the demand of wave attenuation in multiple frequency ranges in engineering. In addition, simplified models for the corresponding edge frequencies of the band gaps are studied, which can contribute to further understanding of the LR mechanism of PCs and the realisation of composite structures with multiple band gaps. The paper is organised as follows. The exact dispersion relations for the propagation of flexural vibrations in infinite Timoshenko beams that are periodically connected with multioscillators are derived in Section 2. The analytical results for the band gaps derived from the new beam are illustrated in Section 3, and the transmission FRFs obtained using the finite element (FE) method are provided to verify the accuracy of the band gap distributions. In Section 4, simplified models are proposed to calculate the initial and terminal frequencies. In addition, the band gaps in the new beam are compared with the beam studied in [24] under different structure parameters. Finally, conclusions are presented in Section 5.

\section{Analytical Models}

In this section, the transfer matrix method is used to derive the exact dispersion relations for the Timoshenko beams with periodically attached multioscillators, which allows for continuity conditions at the two surface boundaries of each unit cell through the use of matrices $[29,30]$. The analytical models of the beams are illustrated in Figure 1. The straight beams extend infinitely along the $x$ axis and have an annular cross section. There are two oscillators assembled on the beam at uniformly spaced intervals, and each oscillator comprises a spring $K$ and a mass $m$. The length $a$ of the interval is called the lattice constant of the PCs. Only flexural vibrations are assumed to occur in the beam. The transverse displacement $y(x, t)$ of the Timoshenko beam satisfies the following equation of motion $[5,31]$ :

$$
\begin{gathered}
\frac{E I}{\rho S} \frac{\partial^{4} y(x, t)}{\partial x^{4}}-\frac{1}{S}\left(1+\frac{E}{\kappa S}\right) \frac{\partial^{4} y(x, t)}{\partial x^{2} \partial t^{2}} \\
+\frac{\partial^{2} y(x, t)}{\partial t^{2}}+\frac{\rho I}{\kappa G S} \frac{\partial^{4} y(x, t)}{\partial t^{4}}=0,
\end{gathered}
$$

where $E$ and $G$ are the Young's modulus and shear modulus of the beam's material, respectively; $\rho$ is the density; $S$ is the cross-sectional area; $\kappa$ is the Timoshenko shear coefficient; and $I$ is the area moment of inertia with respect to the axis perpendicular to the beam axis. By separating out the time variable, $y(x, t)$ can be written as $y(x, t)=Y(x) e^{i \omega t}$, where $\omega$ is the circular frequency. As discussed in [5], for the $n$th cell, 


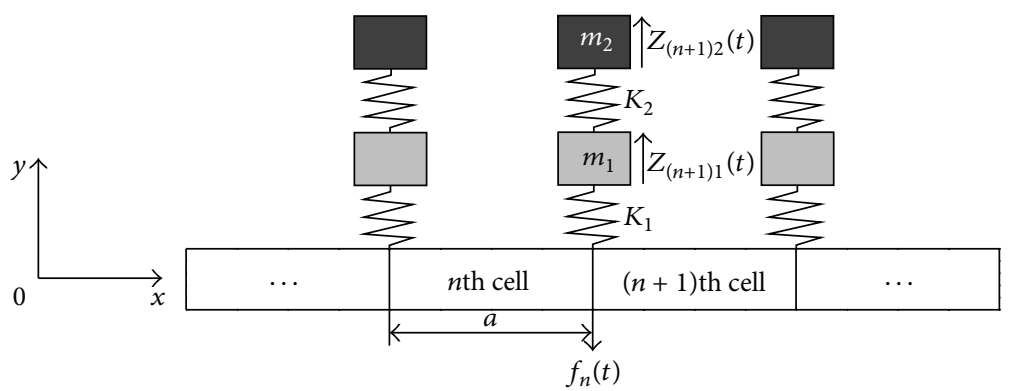

(a)

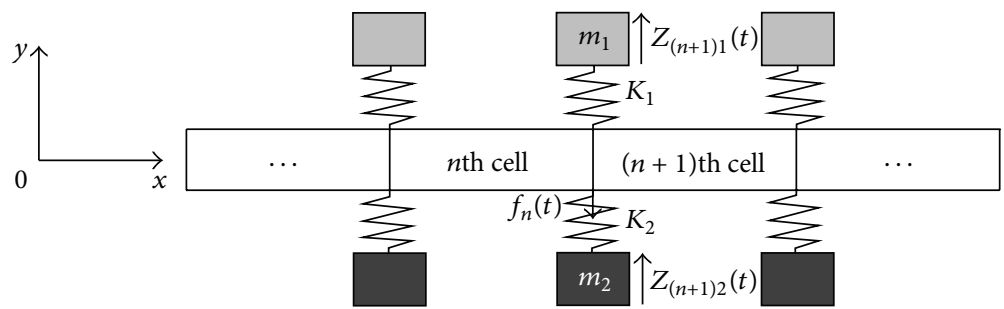

(b)

FIGURE 1: The analytical models of a Timoshenko beam with periodically attached multioscillators. (a) Model A: the oscillators are connected to each other on the same side. (b) Model B: the oscillators are distributed on different sides, a configuration which was put forward and discussed in [24].

where $x^{\prime}=x-n a$, and $n a \leq x \leq(n+1) a$, the amplitude can be obtained by

$$
\begin{aligned}
Y_{n}\left(x^{\prime}\right)= & A_{n} k_{1}^{-3} e^{k_{1} x^{\prime}}+B_{n} k_{2}^{-3} e^{k_{2} x^{\prime}} \\
& +C_{n} k_{3}^{-3} e^{k_{3} x^{\prime}}+D_{n} k_{4}^{-3} e^{k_{4} x^{\prime}},
\end{aligned}
$$

with

$$
k_{j}=(-1)^{[j / 2]} \sqrt{\frac{\alpha+(-1)^{j} \sqrt{\alpha^{2}+4 \beta}}{2}}, \quad \text { for } j=1,2,3,4 \text {, }
$$

where $\alpha=-\rho \omega^{2} / E-\rho \omega^{2} / \kappa G ; \beta=-\rho \omega^{2} / E I-\rho^{2} \omega^{4} / E \kappa G$, and $[j / 2]$ is the largest integer that is less than $j / 2$.

For Model A, which is shown in Figure 1(a), the dynamic equations of the two oscillators connected between the $n$th and $(n+1)$ th cell can be derived as

$$
\begin{aligned}
& m_{1} \ddot{Z}_{(n+1) 1}+K_{1}\left(Z_{(n+1) 1}-y_{n+1}(0, t)\right) \\
& \quad+K_{2}\left(Z_{(n+1) 1}-Z_{(n+1) 2}\right)=0 \\
& m_{2} \ddot{Z}_{(n+1) 2}+K_{2}\left(Z_{(n+1) 2}-Z_{(n+1) 1}\right)=0
\end{aligned}
$$

where $Z_{(n+1) 1}=V_{(n+1) 1} e^{i \omega t}$ and $Z_{(n+1) 2}=V_{(n+1) 2} e^{i \omega t}$ are the displacements of the two oscillators of the $(n+1)$ th cell at the centre of gravity. The absolute values of $V_{(n+1) 1}$ and $V_{(n+1) 2}$ are the vibration amplitudes of the two oscillators of the $(n+$ $1)$ th unit cell. From (4), the relationship between $V_{(n+1) 1}$ and $Y_{(n+1)}(0)$ is

$$
V_{(n+1) 1}=\frac{K_{1} Y_{n+1}(0)}{\left(K_{1}-m_{1} \omega^{2}-K_{2} m_{2} \omega^{2} /\left(K_{2}-m_{2} \omega^{2}\right)\right)} .
$$

The interactive force between the first oscillator and the beam, $F_{n+1}$, at $x^{\prime}=(n+1) a$ is

$$
\begin{aligned}
F_{n+1} & =K_{1}\left[Y_{n+1}(0)-V_{(n+1) A}\right] \\
& =\frac{K_{1} Y_{n+1}(0)\left[m_{1} \omega^{2}+K_{2} m_{2} \omega^{2} /\left(K_{2}-m_{2} \omega^{2}\right)\right]}{\left[m_{1} \omega^{2}+K_{2} m_{2} \omega^{2} /\left(K_{2}-m_{2} \omega^{2}\right)-K_{1}\right]} .
\end{aligned}
$$

For the case of Model B studied in [24], the dynamic equation for the two unconnected oscillators at the interface shown in Figure 1(b) can be derived as [24]

$m_{i} \ddot{Z}_{(n+1) i}+K_{i}\left(Z_{(n+1) i}-y_{n+1}(0, t)\right)=0, \quad i=1,2$.

The solution is

$$
V_{(n+1) i}=\frac{K_{i}}{K_{i}-m_{i} \omega^{2}} Y_{n+1}(0), \quad i=1,2 .
$$

From (8), the interactive force between the oscillators and the beam, $F_{n+1}$, at $x^{\prime}=(n+1) a$ is

$$
\begin{aligned}
F_{n+1} & =\sum K_{i}\left[Y_{n+1}(0)-V_{(n+1) i}\right] \\
& =\sum K_{i}\left(1-\frac{K_{i}}{K_{i}-m_{i} \omega^{2}}\right) Y_{n+1}(0), \quad i=1,2 .
\end{aligned}
$$

According to the continuity of the displacement, slope, bending moment, and shear force at the interface between the $n$th and $(n+1)$ th unit cell, $x^{\prime}=n a$,

$$
\begin{gathered}
Y_{n}(a)=Y_{n+1}(0), \\
Y_{n}^{\prime}(a)=Y_{n+1}^{\prime}(0), \\
E I Y_{n}^{\prime \prime}(a)=E I Y_{n+1}^{\prime \prime}(0), \\
E I Y_{n}^{\prime \prime \prime}(a)=E I Y_{n+1}^{\prime \prime \prime}(0)-F_{n+1} .
\end{gathered}
$$




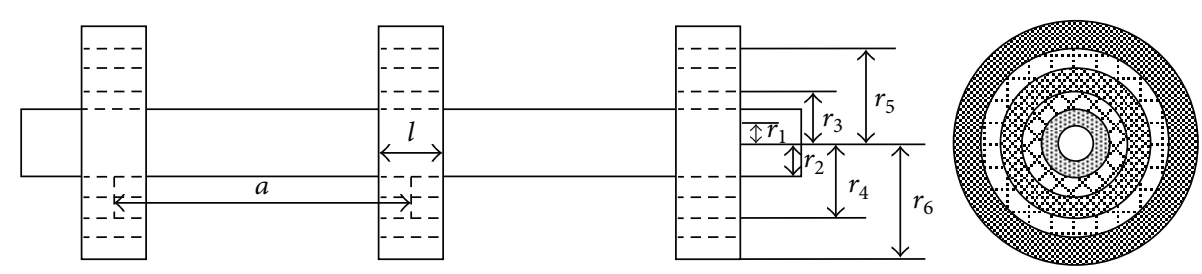

(a)
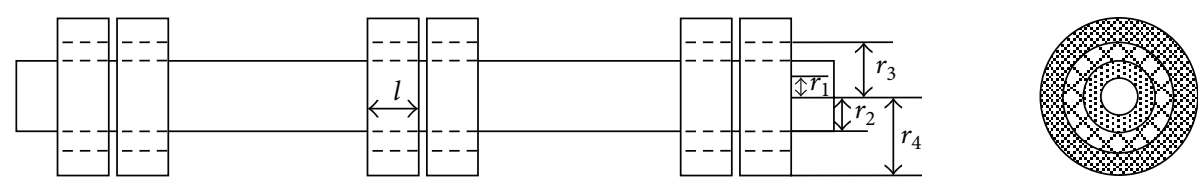

(b)

FIGURE 2: The sketch of a Timoshenko beam with periodically attached multioscillators. (a) Model A, (b) Model B.

By extracting the arbitrary coefficients from (10), $\Phi_{n}=$ $\left[\begin{array}{llll}A_{n} & B_{n} & C_{n} & D_{n}\end{array}\right]^{T}$, these equations can be written in matrix form as follows

$$
\mathbf{M}_{n} \Phi_{n}=\mathbf{N}_{n+1} \Phi_{n+1},
$$

where

$$
\mathbf{M}_{n}=\left[\begin{array}{cccc}
k_{1}^{-3} e^{k_{1} a} & k_{2}^{-3} e^{k_{2} a} & k_{3}^{-3} e^{k_{3} a} & k_{4}^{-3} e^{k_{4} a} \\
k_{1}^{-2} e^{k_{1} a} & k_{2}^{-2} e^{k_{2} a} & k_{3}^{-2} e^{k_{3} a} & k_{4}^{-2} e^{k_{4} a} \\
k_{1}^{-1} e^{k_{1} a} & k_{2}^{-1} e^{k_{2} a} & k_{3}^{-1} e^{k_{3} a} & k_{4}^{-1} e^{k_{4} a} \\
e^{k_{1} a} & e^{k_{2} a} & e^{k_{3} a} & e^{k_{4} a}
\end{array}\right]
$$

$\mathbf{N}_{n+1}$

$$
=\left[\begin{array}{cccc}
k_{1}^{-3} & k_{2}^{-3} & k_{3}^{-3} & k_{4}^{-3} \\
k_{1}^{-2} & k_{2}^{-2} & k_{3}^{-2} & k_{4}^{-2} \\
k_{1}^{-1} & k_{2}^{-1} & k_{3}^{-1} & k_{4}^{-1} \\
1-\frac{F_{n+1} k_{1}^{-3}}{\mathrm{EI}} & 1-\frac{F_{n+1} k_{2}^{-3}}{\mathrm{EI}} & 1-\frac{F_{n+1} k_{3}^{-3}}{\mathrm{EI}} & 1-\frac{F_{n+1} k_{4}^{-3}}{\mathrm{EI}}
\end{array}\right] .
$$

Due to the periodicity of the structure, the Bloch theorem states that

$$
\Phi_{n+1}=e^{i q a} \Phi_{n}
$$

where $q$ is wave vector in the $x$ direction. The problem can be transformed into an eigenvalue matrix equation:

$$
\left|\mathbf{T}-e^{i q a} \mathbf{I}\right|=0
$$

where $\mathbf{T}=\mathbf{N}_{n+1}^{-1} \mathbf{M}_{n}$ and $\mathbf{I}$ is a $4 \times 4$ unit matrix.

The dispersion relation between the wave vector $q$ and the frequency $\omega$ can therefore be obtained. For any $\omega$, if $q$ is a real number, $\omega$ is in the pass band. If $q$ has an imaginary part, the corresponding wave is damped in that region, and the imaginary part of $q$ can be used to describe the attenuation properties in the band gaps.
TABLE 1: Material parameters.

\begin{tabular}{lcccc}
\hline Material & $\begin{array}{c}\text { Density } \\
\left(\mathrm{kg} / \mathrm{m}^{3}\right)\end{array}$ & $\begin{array}{c}\text { Young's } \\
\text { Modulus } E \\
(\mathrm{~Pa})\end{array}$ & $\begin{array}{c}\text { Shear } \\
\text { Modulus } \\
(\mathrm{Pa})\end{array}$ & $\begin{array}{c}\text { Poisson ratio } \\
\sigma\end{array}$ \\
\hline Rubber & 1300 & $7.7 \times 10^{5}$ & $2.6 \times 10^{5}$ & 0.48 \\
$\mathrm{Al}$ & 2600 & $7.0 \times 10^{10}$ & $2.7 \times 10^{10}$ & 0.3 \\
$\mathrm{Cu}$ & 8950 & $1.646 \times 10^{11}$ & $7.53 \times 10^{10}$ & 0.093 \\
Steel & 7780 & $2.106 \times 10^{11}$ & $8.1 \times 10^{10}$ & 0.3 \\
\hline
\end{tabular}

\section{Numerical Simulation and Comparison}

Figure 2 shows two simulation structures of the beam with oscillators based on Model A and Model B, respectively. Both beams are constructed using an aluminium tube, and the oscillators are composed of soft rubber rings and metal rings. As shown in Figure 2(a), the multioscillators in Model A are structures composed of four connected rings in each unit cell, whereas the periodically attached multioscillators in Model B presented in Figure 2(b) are two adjacent structures that are each composed of a rubber ring and a metal ring. The inner and outer radii of the tube are $r_{1}=0.007 \mathrm{~m}$ and $r_{2}=0.01 \mathrm{~m}$, respectively. The lattice constant is $a=0.075 \mathrm{~m}$, and the length of all the rings is $l=0.01 \mathrm{~m}$. The outer radius of the first rubber ring, which is in contact with the tube, is $r_{3}=0.015 \mathrm{~m}$. The outer radius of the first metal ring, which is in contact with the first rubber ring, is $r_{4}=0.0195 \mathrm{~m}$. The radii of the second rubber ring and the metal ring in Figure 2(a) $\left(r_{5}\right.$ and $r_{6}$ ) will be determined in the following

All of the material parameters used in the calculations are listed in the Table 1. As discussed in [32], the shear coefficient of the Timoshenko beam can be determined by

$$
\kappa=\frac{6+12 \sigma+6 \sigma^{2}}{7+12 \sigma+4 \sigma^{2}},
$$

where $\sigma$ denotes the Poisson ratio. 


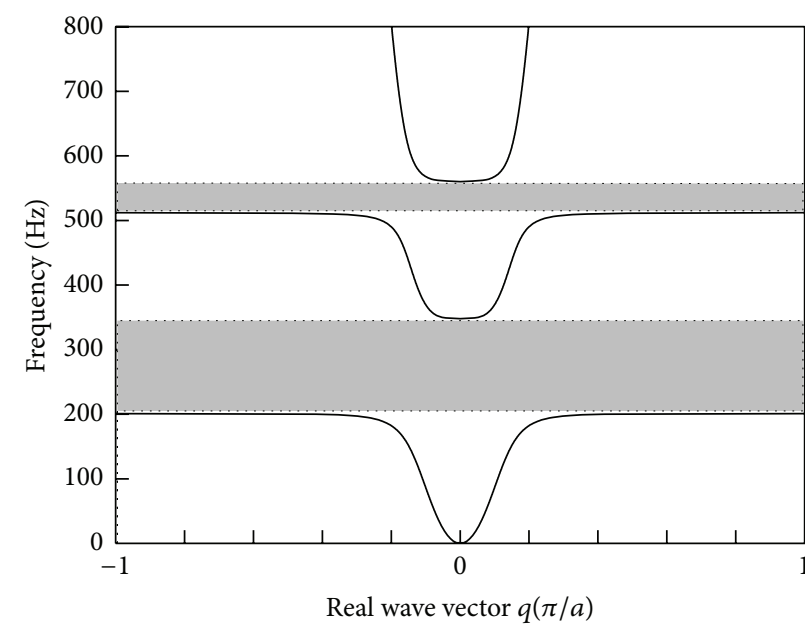

(a)

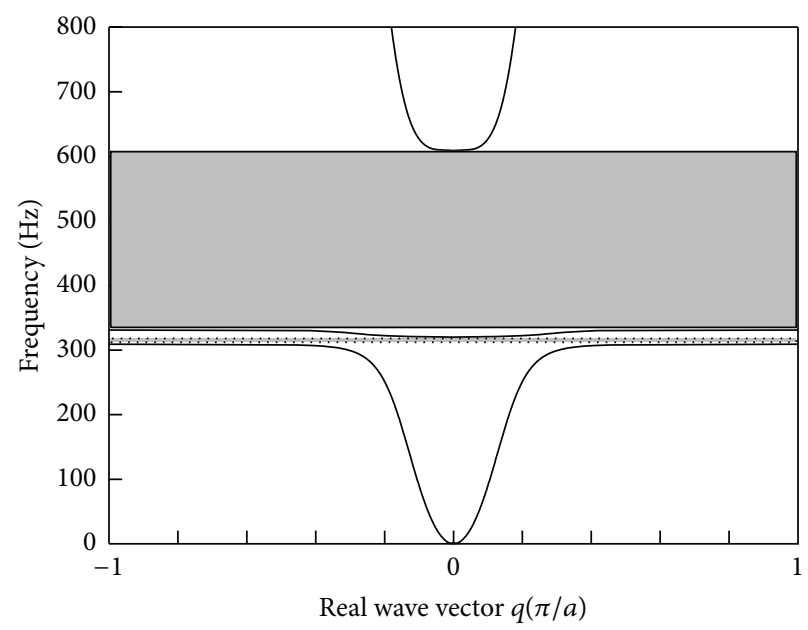

(b)

FIGURE 3: The band structure of the infinite Timoshenko beam with periodically attached multioscillators. (a) Real wave vector of Model A. (b) Real wave vector of Model B.

The radial stiffness of the rubber ring can be calculated using [33]

$$
K=\frac{\pi\left(5+3.29 H^{2}\right) G l}{\ln \left(r_{2} / r_{1}\right)}
$$

where $H=1 /\left(r_{1}+r_{2}\right) \ln \left(r_{1} / r_{2}\right)$ is the shape coefficient.

For comparison, the structure parameters for Model B are taken from [24]. The stiffness and mass of the LR structures in Model A and Model B are the same, namely, $m_{1}=0.0437 \mathrm{~kg}$, $m_{2}=0.0379 \mathrm{~kg}$, and $K_{1}=K_{2}=1.65 \times 10^{5} \mathrm{~N} / \mathrm{m}$. The radii of the second rings are set to $r_{5}=0.02688 \mathrm{~m}$ and $r_{6}=0.02963 \mathrm{~m}$.

The band structures of both models are shown in Figure 3. The complete band gaps are shaded. In both models, two complete band gaps are found between 0 and $800 \mathrm{~Hz}$. The band gap characteristics of Model B based on the Timoshenko beam theory are verified with the results derived in [24]. Compared with the beam with only one oscillator in a unit cell in [5], although the initial frequencies of the first band gaps are both $309 \mathrm{~Hz}$, the total width of the first two band gaps in the Model B is increased from $170.3 \mathrm{~Hz}$ to $297.1 \mathrm{~Hz}$. However, the positions of the first two band gaps are too close and are not suitable for vibration reduction within multiple frequency ranges, especially if the frequency ranges in which vibration must be reduced have large intervals.

Figure 3(a), obtained using Model A, shows two widely separated band gaps that are not obtained in Model B. The initial frequency of the first gap in Model A is decreased to $201.4 \mathrm{~Hz}$. Note that the centre frequency of the second band gap $(511.8-560.9 \mathrm{~Hz})$ is approximated two times the centre frequency of the first gap $(201.4-348.4 \mathrm{~Hz})$; that is, a frequency-multiplication relationship between the two resonance-type gap can be achieved. The "frequencymultiplication relationship" mentioned here can be explained as follows: the central frequency of the band gaps is close to the frequency $f_{N}=N \times f_{1}(N$ could be $1,2,3, \ldots)$, where the fundamental frequency $f_{1}$ is the central frequency of the first band gap. Thus, the flexural vibrations at one and two times the fundamental frequency can be well reduced, a phenomenon that can be employed to address a case such as the above-mentioned angular misalignment of the coupling in a rotor system. In addition, the first band gap in Model A is wider than the second band gap, which helps to flexibly reduce vibrations of the beam at low frequencies.

The existence of the band gaps calculated from the infinite system can be verified by the transmission property derived from a corresponding finite system because PCs with a sufficient number of unit cells can provide a large wave attenuation in the corresponding band gap range [11]. The FRF, which represents the relationship between the wave response and the corresponding frequencies, has been used to describe vibration gaps effectively. Therefore, the finite system of Model A is created in Abaqus to calculate the FRF. The mesh model for the FE method is illustrated in Figure 4(a), which has the same geometry in the unit cell as the model in Figure 2(a). Based the analysis of different numbers of unit cells in the structure [11], eight unit cells used in an FE simulation can achieve a sufficient accuracy for approximating the results of the infinite system. Therefore, the length of the beam is $0.6 \mathrm{~m}$ in Figure 4(a). To guarantee the free vibration of the beam, there are no boundary constraints at the ends of the beam. The acceleration is induced at the left end of the beam in the $y$ direction, and the corresponding acceleration is extracted at the opposite end. The frequency responses are illustrated as solid lines in Figure 4(b).

Note the two sharp drops below the $0 \mathrm{~dB}$ line (dashed line) in the Figure 4(b), which indicate the ranges of the band gaps. Compared with the response outside the band gaps, the average response attenuation of the two band gaps is approximately $40 \mathrm{~dB}$. The ranges of the first two gaps calculated using the FE method are similar to those of the gaps shown in Figure 3(a), which are obtained from the 


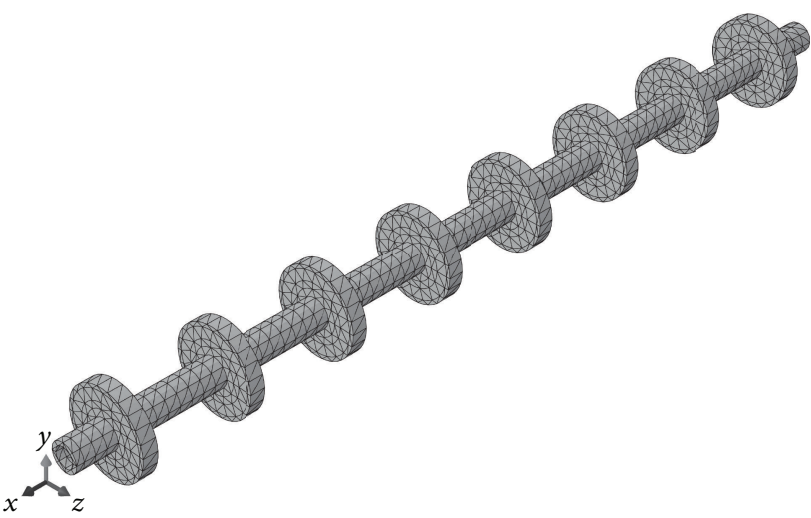

(a)

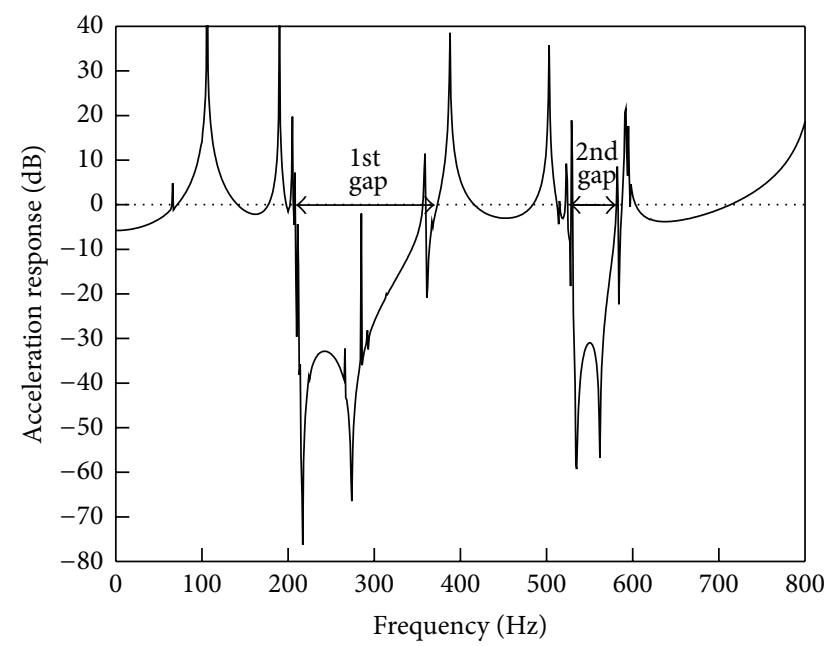

(b)

FIGURE 4: (a) The calculation model for the FE method. (b) The corresponding calculated FRF.

infinite structure. It can be concluded that the previous analysis is accurate, and using LR structures with equivalent parameters, Model A has better band gaps for vibration reduction in the frequency-multiplication ranges than does Model B. Considering the advantages of each model, an LR PC beam with the desired band gap properties can be obtained by choosing the most appropriate plan.

\section{Simplified Models for the Edge Frequencies of Band Gaps}

In this section, the corresponding simplified models for the initial and terminal frequencies of the band gaps for Model A are studied. The simplified models for Model B have been discussed previously in [24].

4.1. Initial Frequency Model. The initial frequency of the first band gap in a typical LR PC is determined by the resonance frequency of the oscillator in the same direction. In this resonance mode, the oscillators vibrate in specific directions, and the phases of the oscillator vibrations in adjacent unit cells are reversed to keep the dynamic balance $[4,24]$. Thus, the simplified model for the initial frequencies of the two oscillators can be formed as shown in Figure 5 .

The equations of motion for the model are as follows:

$$
\begin{gathered}
m_{1} \ddot{u}_{1}+K_{1} u_{1}+K_{2}\left(u_{1}-u_{2}\right)=0, \\
m_{2} \ddot{u}_{2}+K_{2}\left(u_{2}-u_{1}\right)=0,
\end{gathered}
$$

where $u_{1}$ and $u_{2}$ represent the displacements of the respective oscillator.

The natural angular frequency $\omega_{i n}$ satisfies the equation

$$
\operatorname{det}\left[\mathbf{k}-\omega_{\text {in }}^{2} \mathbf{m}\right]=0 \text {, }
$$

where $\mathbf{k}=\left[\begin{array}{cc}K_{1}+K_{2} & -K_{2} \\ -K_{2} & K_{2}\end{array}\right], \mathbf{m}=\left[\begin{array}{ll}m_{1} & \\ & m_{2}\end{array}\right]$.

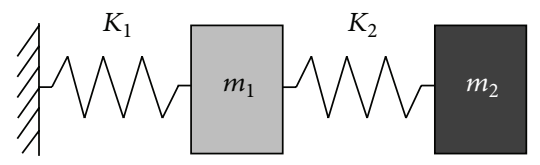

FIGURE 5: The simplified model for the initial frequencies of band gaps.

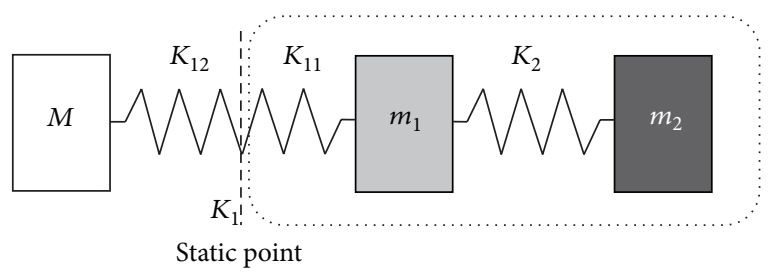

FIgURE 6: The simplified model for the terminal frequencies of the band gaps.

Thus,

$$
\begin{aligned}
\omega_{i 1,2}^{2}= & \frac{K_{1} m_{2}+K_{2} m_{1}+K_{2} m_{2}}{2 m_{1} m_{2}} \\
& \mp \frac{\sqrt{\left(K_{1} m_{2}+K_{2} m_{1}+K_{2} m_{2}\right)^{2}-4 K_{1} K_{2} m_{1} m_{2}}}{2 m_{1} m_{2}}
\end{aligned}
$$

can be obtained, and the initial frequencies of the first two band gaps are $f_{i 1,2}=\sqrt{\omega_{i 1,2}} / 2 \pi$.

4.2. Terminal Frequency Model. All of the oscillation phases of the unit cells are in the same direction at the terminal frequency of the band gap. The dynamic balance is given by the antiphases between the LR structures and the matrix [4, 24]. The matrix mentioned in $[4,24]$ is the beam in this paper by analogy. As illustrated in Figure 6, the simplified model for 


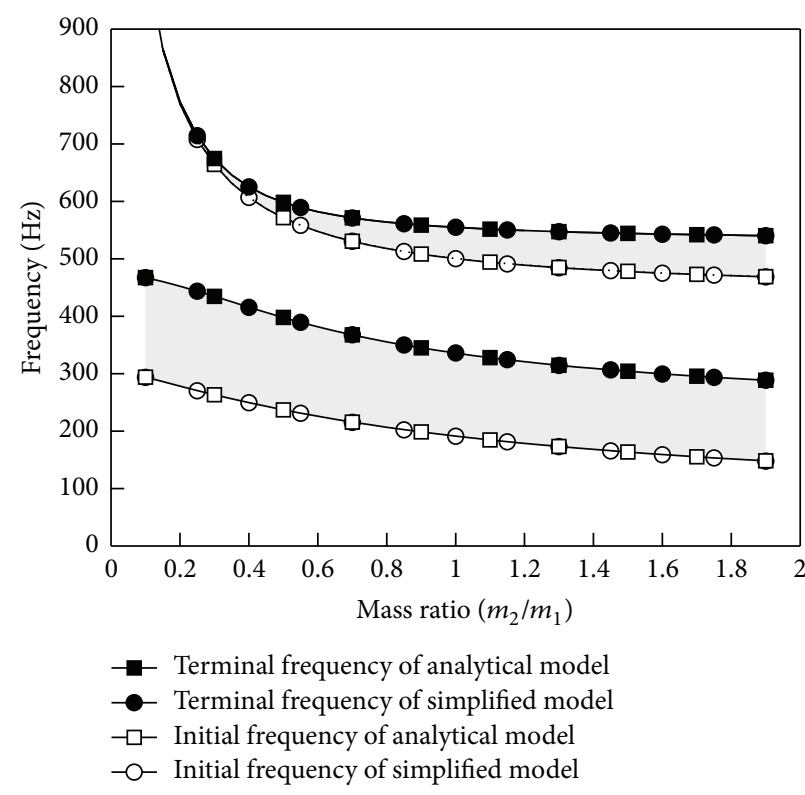

(a)

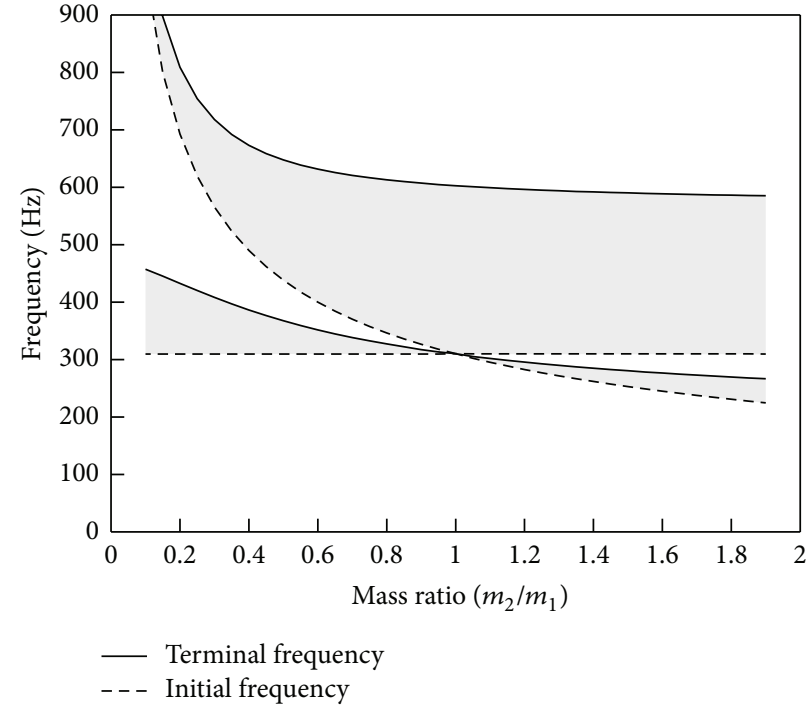

(b)

Figure 7: Variation of the band gaps as a function of the oscillators' mass ratio and verification of the simplified models. (a) Model A, (b) Model B. $\left(m_{1}=0.0437 \mathrm{~kg}, M=0.0312 \mathrm{~kg}\right.$, and $\left.K_{1}=K_{2}=1.65 \times 10^{5} \mathrm{~N} / \mathrm{m}\right)$.

the terminal frequencies comprises the beam mass $M$ and the oscillators in the unit cell. There is a static point between the beam and the connected oscillators that divides the model into two parts that have the same natural frequencies. At the static point, the spring $K_{1}$ can be considered a series connection of two springs $K_{11}$ and $K_{12}$, which are related by the following equation:

$$
\frac{1}{K_{1}}=\frac{1}{K_{11}}+\frac{1}{K_{12}}
$$

The components to the right of the static point (dashed box) can be observed as a single unit. The natural angular frequency $\omega_{t n}$ is described by (17) to (19), where $K_{1}$ is replaced by $K_{11}$.

Because the resonances of the matrix and the connected oscillators are at the same frequency,

$$
\frac{K_{12}}{M}=\omega_{t n}
$$

Thus, the relation between $K_{11}$ and $\omega_{t n}$ can be extracted and combined with the previous discussion in Section 4.1. Therefore,

$$
\begin{aligned}
\omega_{t 1,2}^{2}= & \frac{M K_{1} m_{2}+M K_{2} m_{1}+M K_{2} m_{2}+K_{1} m_{1} m_{2}}{2 M m_{1} m_{2}} \\
& \mp \frac{\sqrt{\left(M K_{1} m_{2}+M K_{2} m_{1}+M K_{2} m_{2}-K_{1} m_{1} m_{2}\right)^{2}+4 M K_{1} m_{1} m_{2}\left(K_{1} m_{2}-M K_{2}\right)}}{2 M m_{1} m_{2}},
\end{aligned}
$$

and the terminal frequencies of the first two band gaps are $f_{t 1,2}=\sqrt{\omega_{t 1,2}} / 2 \pi$.

Figures 7, 8, and 9 illustrate the dependence of the band gaps on the oscillators' mass ratio, the oscillators' stiffness ratio, and the beam's mass, respectively. In addition, for Model $A$, the calculations of the initial and terminal frequencies of the first two band gaps using both the analytical and simplified models are presented to verify the accuracy of the deduced formulae. The shadow regions indicate the band gaps, and the details of the data are illustrated in the top right corner of each figure.

Figures 7(a), 8(a), and 9(a) show that the frequencies of the band gaps obtained using the simplified models and the analytical model are in good agreement. These results prove the accuracy and validity of the methods proposed in this paper. The beams with periodically attached multioscillators have similar resonance modes to those of typical LR PCs at the boundary frequencies of the band gaps. This result reveals 


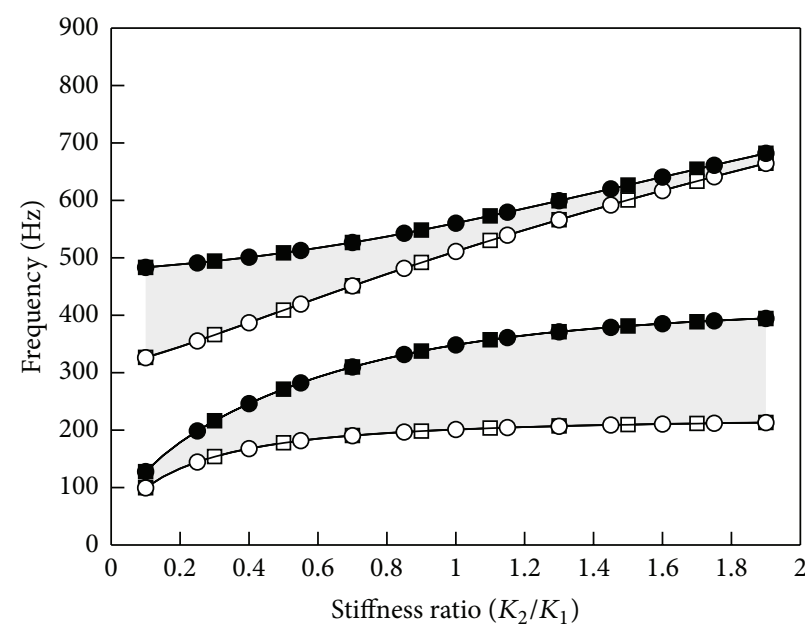

- Terminal frequency of analytical model

- Terminal frequency of simplified model

$-\square-$ Initial frequency of analytical model

-O- Initial frequency of simplified model

(a)

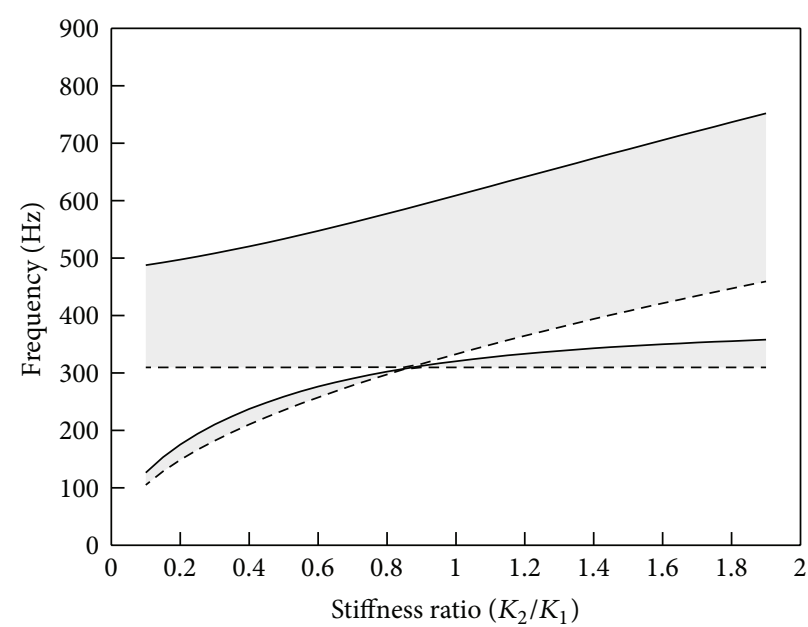

- Terminal frequency

- - - Initial frequency

(b)

FIGURE 8: Variation of the band gaps as a function of the oscillators' stiffness ratio and verification of the simplified models. (a) Model A, (b) Model B. $\left(m_{1}=0.0437 \mathrm{~kg}, m_{2}=0.0379 \mathrm{~kg}, M=0.0312 \mathrm{~kg}\right.$, and $\left.K_{1}=1.65 \times 10^{5} \mathrm{~N} / \mathrm{m}\right)$.

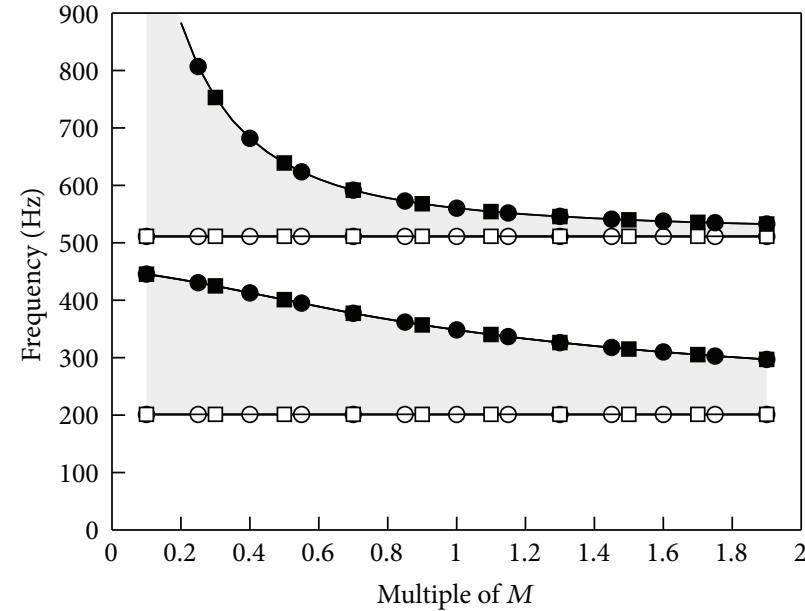

- Terminal frequency of analytical model

- Terminal frequency of simplified model

$-\square-$ Initial frequency of analytical model

-0 Initial frequency of simplified model

(a)

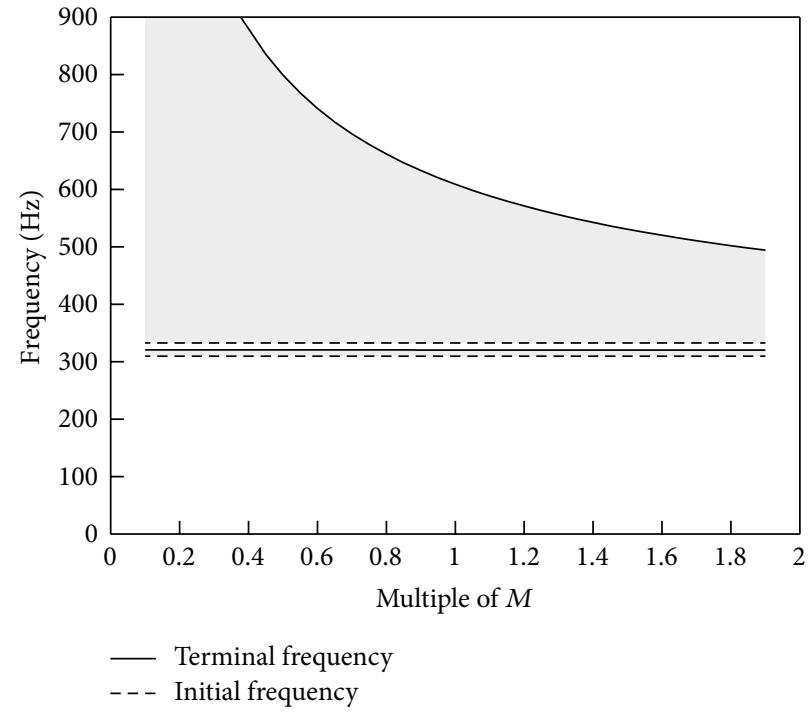

(b)

Figure 9: Variation of the band gaps as a function of the beam's mass and verification of the simplified models. (a) Model A, (b) Model B. $\left(m_{1}=0.0437 \mathrm{~kg}, m_{2}=0.0379 \mathrm{~kg}, M=0.0312 \mathrm{~kg}\right.$, and $\left.K_{1}=K_{2}=1.65 \times 10^{5} \mathrm{~N} / \mathrm{m}\right)$.

the characteristics of the LR mechanism, which is helpful in the construction of new devices with LR band gaps.

By comparing the (a) subfigure with the (b) subfigure in Figures 7-9, it can be seen that the variation tendencies of Model A are similar to those of Model B. With the increase of the oscillators' mass as well as the beam's mass or with the reduction of the oscillators' stiffness, the frequencies of the band gaps are decreased. However, there is usually a distance between the first two band gaps in Model A, and the band gaps that are widely separated can be obtained without large differences in the parameters of the oscillators. Thus, Model A has better regulation and control abilities in practical engineering. In addition, with the same material parameters, Model A clearly always has a lower initial 
frequency and a wider first band gap than Model B and is able to provide a larger range of vibration reduction at low frequencies. To achieve lower frequencies, Model B should use a larger mass or a smaller stiffness, which is uneconomic in most engineering. In addition, although the total width of the gaps is larger, a narrow first band gap is almost inevitable in Model B. Furthermore, the band gaps with a frequency-multiplication relationship cannot be well derived in Model B; thus, this model is not appropriate for vibration damping or noise reduction in the frequency-multiplication ranges. In general, Model A has specific abilities and can be more reasonably and feasibly applied to practical structures because of the advantages of lower frequencies, frequencymultiplication relationships, and material costs.

\section{Conclusions}

In this paper, a new Timoshenko beam structure with periodically attached multioscillators is proposed to obtain band gaps in the frequency-multiplication ranges based on the LR mechanism of PCs. Explicit matrix formulations are derived for the calculation of wave band structures of the new beam by using the transfer matrix method. The gap characteristics of the beam are confirmed by calculating the FRF of the corresponding finite structure. The numerical calculations of the band structures and the analysis of the model parameters demonstrate that the beams with periodically attached multioscillators have more abundant gap characteristics than those with only one oscillator in a unit cell. By using common materials and an uncomplicated beam structure, multiple resonance-type band gaps with large wave-attenuation and frequency-multiplication ranges, together with the wider and lower first band gap, are derived in the new beam; this result was not illustrated in any of the previous studies on LR PC beams. In addition, simplified models are proposed to deduce accurate estimation formulae for the initial and terminal frequencies of the band gaps in the new beam. The simplified models will also contribute to enhanced understanding of the LR mechanism of PCs and will facilitate the analysis of similar structures.

The research findings presented in this paper provide suggestions for future studies of small-size PCs with low frequencies and multiple resonance-type band gaps. Moreover, the results can be employed to create new devices that reduce vibration and mitigate noise in the frequency-multiplication ranges for aerospace and civil structures.

\section{Acknowledgment}

This work is supported by the National Nature Science Foundation of China under Grant nos. 51079127, 51179171, and 51279180 .

\section{References}

[1] E. I. Rivin, Passive Vibration Isolation, ASME Press, New York, NY, USA, 2003.
[2] M. M. Sigalas and E. N. Economou, "Elastic and acoustic wave band structure," Journal of Sound and Vibration, vol. 158, no. 2, pp. 377-382, 1992.

[3] M. S. Kushwaha, P. Halevi, L. Dobrzynski, and B. DjafariRouhani, "Acoustic band structure of periodic elastic composites," Physical Review Letters, vol. 71, no. 13, pp. 2022-2025, 1993.

[4] X. S. Wen, J. H. Wen, D. L. Yu et al., Phononic Crystals, National Defense, Industry Press, Beijing, China, 2009.

[5] D. Yu, Y. Liu, G. Wang, H. Zhao, and J. Qiu, "Flexural vibration band gaps in Timoshenko beams with locally resonant structures," Journal of Applied Physics, vol. 100, no. 12, Article ID 124901, 2006.

[6] J. H. Sun, C. W. Lan, C. Y. Kuo, and T. T. Wu, "A ZnO/silicon Lamb wave filter using phononic crystals," in Proceedings of the IEEE International Frequency Control Symposium, pp. 1-4, Baltimore, Md, USA, May 2012.

[7] K. M. Ho, C. K. Cheng, Z. Yang, X. X. Zhang, and P. Sheng, "Broadband locally resonant sonic shields," Applied Physics Letters, vol. 83, no. 26, pp. 5566-5568, 2003.

[8] Z. Liu, X. Zhang, Y. Mao et al., "Locally resonant sonic materials," Science, vol. 289, no. 5485, pp. 1734-1736, 2000.

[9] C. Goffaux, J. Sánchez-Dehesa, A. L. Yeyati et al., "Evidence of Fano-like interference phenomena in locally resonant materials," Physical Review Letters, vol. 88, no. 22, Article ID 225502, 4 pages, 2002.

[10] P. Sheng, X. X. Zhang, Z. Liu, and C. T. Chan, "Locally resonant sonic materials," Physica B, vol. 338, no. 1-4, pp. 201-205, 2003.

[11] J. S. Jensen, "Phononic band gaps and vibrations in one- and two-dimensional mass-spring structures," Journal of Sound and Vibration, vol. 266, no. 5, pp. 1053-1078, 2003.

[12] M. Hirsekorn, "Small-size sonic crystals with strong attenuation bands in the audible frequency range," Applied Physics Letters, vol. 84, no. 17, pp. 3364-3366, 2004.

[13] G. Wang, D. Yu, J. Wen, Y. Liu, and X. Wen, “One-dimensional phononic crystals with locally resonant structures," Physics Letters A, vol. 327, no. 5-6, pp. 512-521, 2004.

[14] Z. Liu, C. T. Chan, and P. Sheng, "Analytic model of phononic crystals with local resonances," Physical Review B, vol. 71, no. 1, Article ID 014103, 8 pages, 2005.

[15] H. H. Huang and C. T. Sun, "Wave attenuation mechanism in an acoustic metamaterial with negative effective mass density," New Journal of Physics, vol. 11, Article ID 013003, 2009.

[16] H. H. Huang, C. T. Sun, and G. L. Huang, "On the negative effective mass density in acoustic metamaterials," International Journal of Engineering Science, vol. 47, no. 4, pp. 610-617, 2009.

[17] G. Wang, X. Wen, J. Wen, and Y. Liu, "Quasi-one-dimensional periodic structure with locally resonant band gap," Journal of Applied Mechanics, vol. 73, no. 1, pp. 167-170, 2006.

[18] D. Yu, Y. Liu, G. Wang, L. Cai, and J. Qiu, "Low frequency torsional vibration gaps in the shaft with locally resonant structures," Physics Letters A, vol. 348, no. 3-6, pp. 410-415, 2006.

[19] Y. Xiao, J. H. Wen, and X. S. Wen, "Longitudinal wave band gaps in metamaterial-based elastic rods containing multi-degreeof-freedom resonators," New Journal of Physics, vol. 14, no. 3, Article ID 033042, 2012.

[20] D. Yu, Y. Liu, H. Zhao, G. Wang, and J. Qiu, "Flexural vibration band gaps in Euler-Bernoulli beams with locally resonant structures with two degrees of freedom," Physical Review B, vol. 73 , no. 6, pp. 1-5, 2006. 
[21] Y. Liu, D. Yu, L. Li, H. Zhao, J. Wen, and X. Wen, "Design guidelines for flexural wave attenuation of slender beams with local resonators," Physics Letters A, vol. 362, no. 5-6, pp. 344347, 2007.

[22] L. Liu and M. I. Hussein, "Wave motion in periodic flexural beams and characterization of the transition between Bragg scattering and local resonance," Journal of Applied Mechanics, vol. 79, no. 1, Article ID 011003, 17 pages, 2012.

[23] Y. Xiao, J. H. Wen, and X. S. Wen, "Broadband locally resonant beams containing multiple periodic arrays of attached resonators," Physics Letters A, vol. 376, no. 16, pp. 1384-1390, 2012.

[24] Q. H. Wen, S. G. Zuo, and H. Wei, "Locally resonant elastic wave band gaps in flexural vibration of multi-oscillators beam," Acta Physica Sinica, vol. 61, no. 3, Article ID 034301, 2012.

[25] D. Yu, J. Wen, H. Zhao, Y. Liu, and X. Wen, "Vibration reduction by using the idea of phononic crystals in a pipe-conveying fluid," Journal of Sound and Vibration, vol. 318, no. 1-2, pp. 193-205, 2008.

[26] M. Oudich, Y. Li, B. M. Assouar, and Z. Hou, "A sonic band gap based on the locally resonant phononic plates with stubs," New Journal of Physics, vol. 12, Article ID 083049, 2010.

[27] J. C. Hsu, "Local resonances-induced low-frequency band gaps in two-dimensional phononic crystal slabs with periodic stepped resonators," Journal of Physics D, vol. 44, no. 5, Article ID 055401, 2011.

[28] Y. Xiao, J. H. Wen, and X. S. Wen, "Flexural wave band gaps in locally resonant thin plates with periodically attached springmass resonators," Journal of Physics D, vol. 45, no. 19, Article ID 195401, 2012.

[29] W. T. Thomson, "Transmission of elastic waves through a stratified solid medium," Journal of Applied Physics, vol. 21, no. 2, pp. 89-93, 1950.

[30] R. Esquivel-Sirvent and G. H. Cocoletzi, "Band structure for the propagation of elastic waves in superlattices," Journal of the Acoustical Society of America, vol. 95, no. 1, pp. 86-90, 1994.

[31] R. A. Méndez-Sánchez, A. Morales, and J. Flores, "Experimental check on the accuracy of Timoshenko's beam theory," Journal of Sound and Vibration, vol. 279, no. 1-2, pp. 508-512, 2005.

[32] T. Kaneko, "On Timoshenko's correction for shear in vibrating beams," Journal of Physics D, vol. 8, no. 16, pp. 1927-1936, 1975.

[33] C. S. Zhao and S. J. Zhu, "Study on the static stiffness characteristics of rubber-metal ring," China Mechanical Engineering, vol. 15, no. 11, pp. 962-964, 2004. 


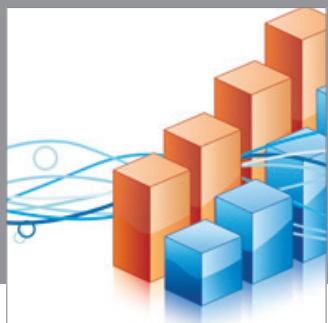

Advances in

Operations Research

mansans

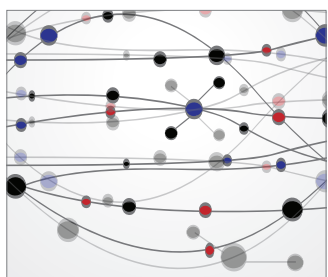

The Scientific World Journal
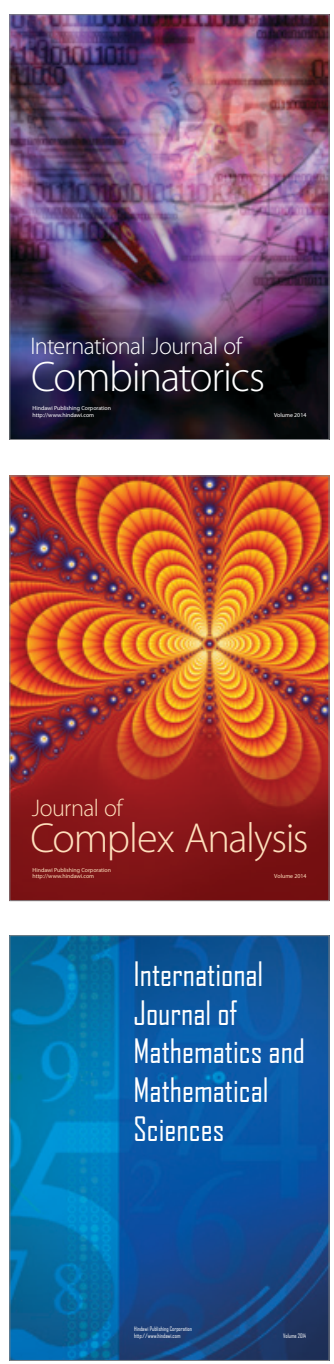
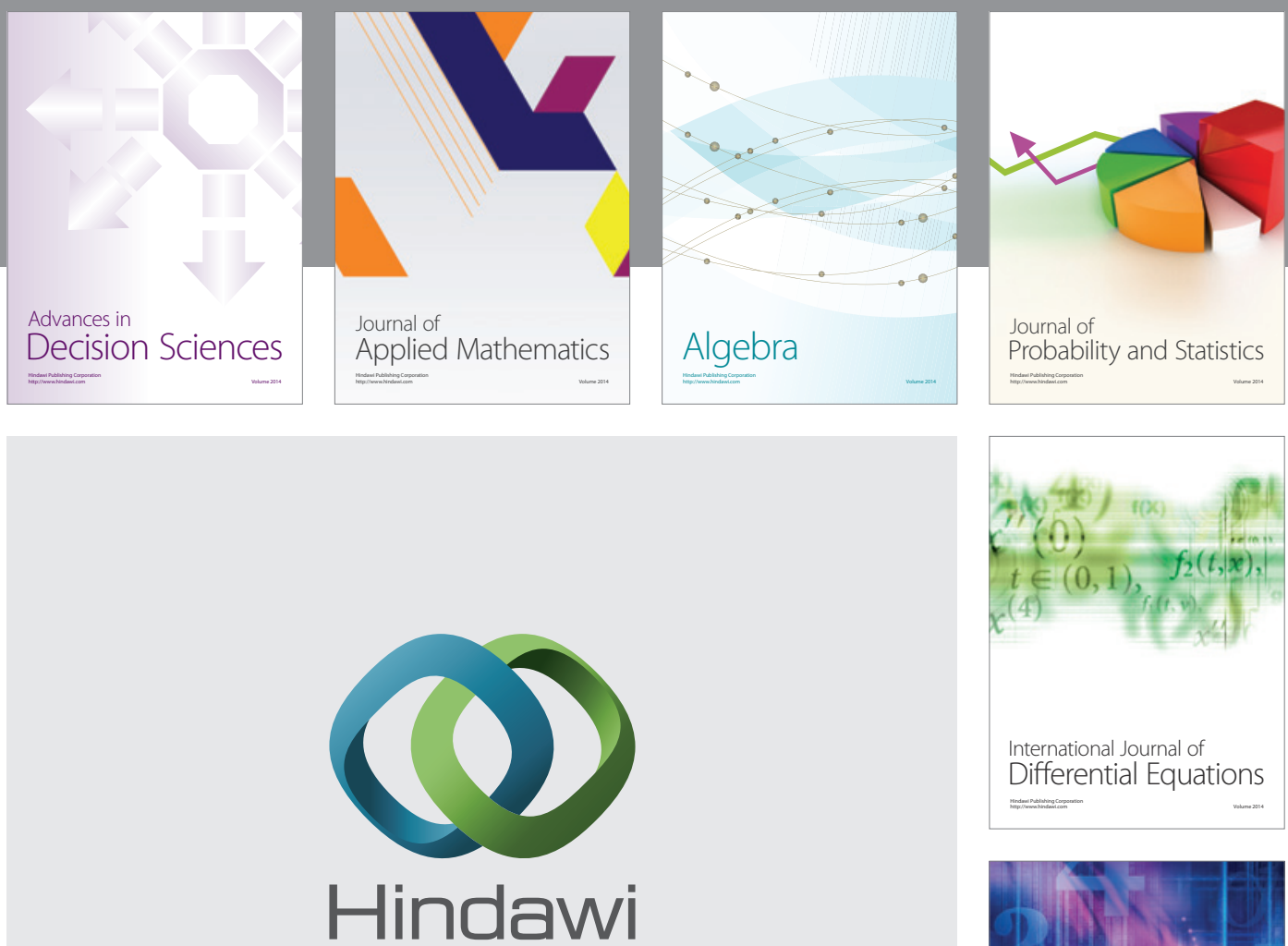

Submit your manuscripts at http://www.hindawi.com
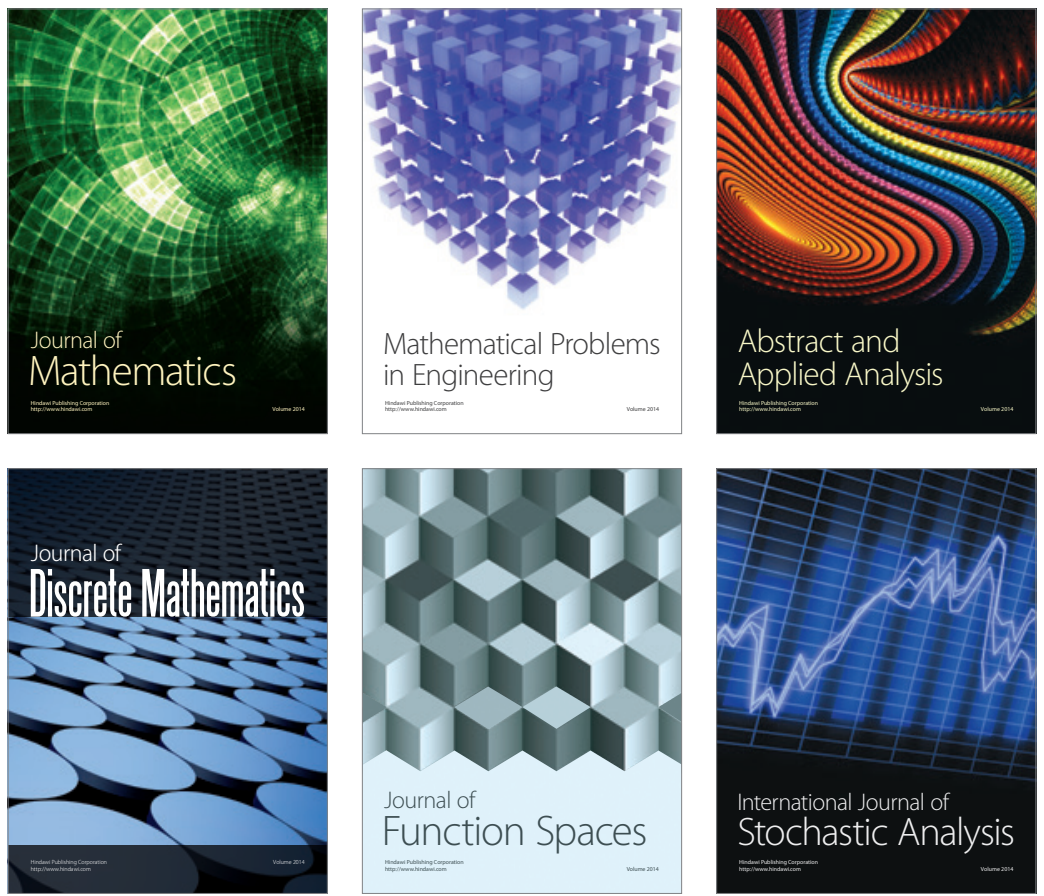

Journal of

Function Spaces

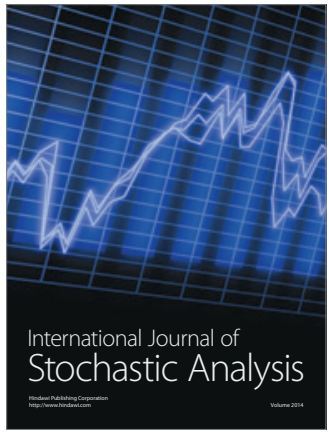

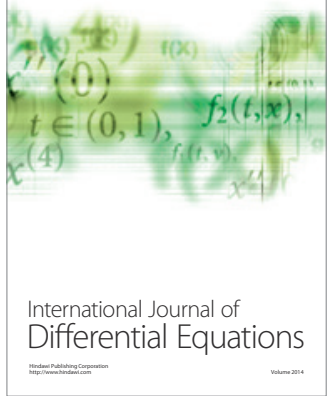
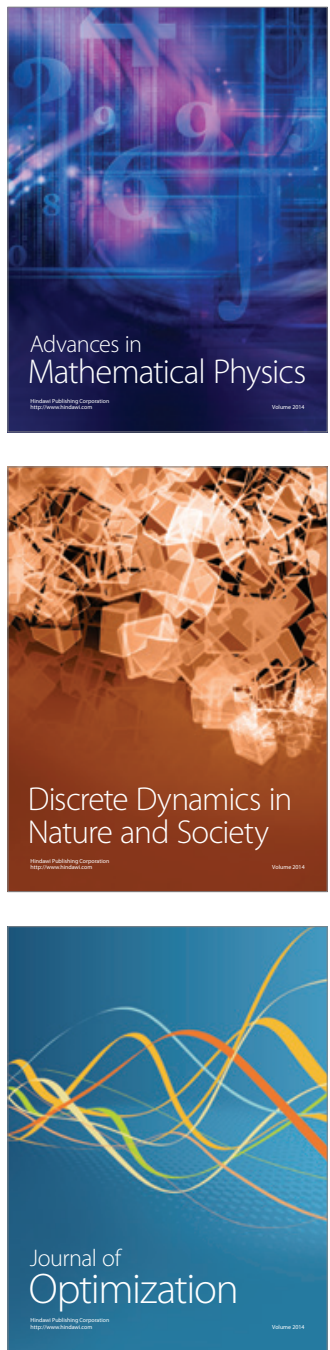